\title{
Protein domains, catalytic activity, and subcellular distribution of mouse NTE-related esterase
}

\author{
Ping'an Chang $\cdot$ Zhan-xiang Wang • \\ Dingxin Long $\cdot$ Wenzhen Qin $\cdot$ Yijun Wu
}

Received: 11 March 2009/Accepted: 9 June 2009/Published online: 23 June 2009

(C) Springer Science+Business Media, LLC. 2010

This article has been withdrawn due to a disagreement between authors and the original laboratory where the research was conducted.

This article has been withdrawn due to a disagreement between authors and the original laboratory where the research was conducted.

P. Chang $(\bowtie) \cdot$ W. Qin

Key Laboratory of Molecular Biology,

College of Bio-information, Chongqing University of Posts

and Telecommunications, Chongqing, Nan'an District 400065,

People's Republic of China

e-mail: changpingan@yahoo.com.cn

P. Chang $\cdot$ D. Long $\cdot$ Y. Wu

Laboratory of Molecular Toxicology, Institute of Zoology,

Chinese Academy of Sciences, Beijing 100080, People's

Republic of China

Z. Wang

Department of Neurosurgery, The First Hospital of Xiamen City,

Xiamen 361001, People's Republic of China

D. Long

School of Public Health, University of South China,

Hengyang 421001, People's Republic of China 